\title{
Research on the Ergonomic Design of Pet Dog Toothbrush Neck
}

\author{
Yu Shu Huang*,a, Cheng Qi Xue ${ }^{2, b}$ \\ ${ }^{1}$ Mechanical Engineering College Southeast University Nanjing, China \\ ${ }^{2}$ Mechanical Engineering College Southeast University Nanjing, China
}

\begin{abstract}
Using a toothbrush to clean the teeth for a pet dog is the best way to maintain the pet's oral health. Meanwhile, the angle of a toothbrush neck can influence the posture and brushing strength of the pet caretaker, thereby affecting the cleaning efficiency. In order to compare the cleaning efficiency of pet dog toothbrushes with varied neck types, 20 volunteers with dog caring experience were selected. A medium-sized dog tooth model coated with water soluble pigment was provided to each volunteer, and then she/he was asked to brush the model for three minutes, using a specific type of pet dog toothbrush. Such process was repeated for each of 5 toothbrushes with varied neck types (all other elements remained the same), while different neck types were provided to each volunteer in random sequence. The pigment areas of the incisors, canines, and molars before and after tooth brushing were measured and calculated to speculate the cleaning efficiency of the toothbrush. At the end of the experiment, each subject was asked to evaluate five toothbrushes with respect to operating comfortability. The experimental results showed that the toothbrushes with backward tilting angle at the upper section of their brush necks performed significantly better than the other four groups of toothbrushes in cleaning molar and canine teeth, while the toothbrushes with backward tilting angle at the lower section, and with the upper section parallel to the brush handle were more comfortable in operation.
\end{abstract}

\section{Introduction}

Food debris will form dental plaque in the oral cavity of pet dogs. If it is not well cleaned, the dental plaque will begin to calcify and become dental calculus in 48 hours, which will not only lead to halitosis, but may even cause oral diseases, such as gingivitis and periodontal disease [1]. According to Studies by R. M. Allan [2] have shown that to maintain the health of pet dog teeth, cleaning teeth with a toothbrush is the most effective way compared with prescription dentifrice-food and dentifrice chewing biscuits, because physical friction and swing of the toothbrush bristles with the tooth surface can avoid the generation of dental calculus.

The detailed design of the toothbrush will affect the clinical effect of tooth cleaning. There have been many related studies on human toothbrushes. According to the current research literature on toothbrushes, it is known that the bristles and brush necks are the main factors affecting the cleaning rate of teeth. [3] Many scholars have studied the relationship between various parameters of bristles affecting tooth cleaning rate, including the density of bristles [4], the arrangement of bristles [5,6], the hardness of bristles [7], the material of bristles [8], etc. Some scholars have also conducted research on the angle design of toothbrushes [9] and found that the angle design of toothbrushes affects the way people brush their teeth, thereby affecting the cleaning rate of teeth.

On the other hand, the design of the toothbrush needs to consider the comfort of operation. Guang. Cheng et al.

*a2320765290@qq.com

bipd_xcq@seu.edu.cn
[10] established a new man-machine model between the upper limbs and the toothbrush, analyzed the actions and rules of people's daily brushing, and proved that the length and curvature of the toothbrush will affect people's posture when brushing, which laid the foundation for brushing. The basic relationship between product design and toothbrush design structure. Zhu, Y. F. et al. [11] studied the general design principles of toothbrush handles from an ergonomic point of view. Based on a large amount of literature, it can be known that for the design of a human toothbrush, a toothbrush with a small brush head, suitable curvature, suitable handle length and grip shape can maximize the comfort of use.

The design factors that affect tooth cleaning rate and operating comfort are sorted out and drawn into Figure 1. Due to the lack of relevant research on the design of dog toothbrushes, it can be speculated that these design factors will also affect the owner's tooth cleaning rate and operating comfort when brushing the dog's teeth. However, human toothbrushes are suitable for the oral structure of humans, while canine mouth shape and tooth shape are very different from humans: canines have a long snout and adult dogs have 42 permanent teeth. There were 20 teeth in maxilla and 22 in the lower jaw. The maxilla consists of 6 incisors, 2 canine teeth, and 12 molar teeth, and the the lower jaw is the same as the maxilla except that there are 2 more molar teeth. Dogs' teeth are mainly used for tears. The shape of canine teeth is trapezoidal, unlike human teeth, which are arcuate. Furthermore, in adult dogs, there is often a lack of two molars (upper and lower), so the teeth 
are separated and there is a gap between the teeth that easily accumulates debris. In contrast to human teeth, canine teeth have posterior crowns with sharp tips as sharp ridges that used to tear surfaces. Due to these differences, the canine toothbrush design should be different from the human toothbrush, and the design should be adapted to the configuration of the canine jaw and the spatial relationship of the teeth.

Furthermore, the posture of people brushing their pet dogs' teeth is obviously different from the posture of brushing their own teeth. The relative position of teeth and upper limbs changes, and people's brushing postures and movement methods will also change accordingly.

Based on the above literature, combined with the observation of the actual situation of the owner brushing the teeth of the pet dog, the author of this article believes that if the shape and design of the brush neck is changed, the rear teeth of the dog can be cleaned, and the teeth can be aligned more accurately, and the owner can use it. More comfortable. Therefore, this study uses five toothbrushes with different neck styles and the same specifications to compare the cleaning rate and operating comfort.

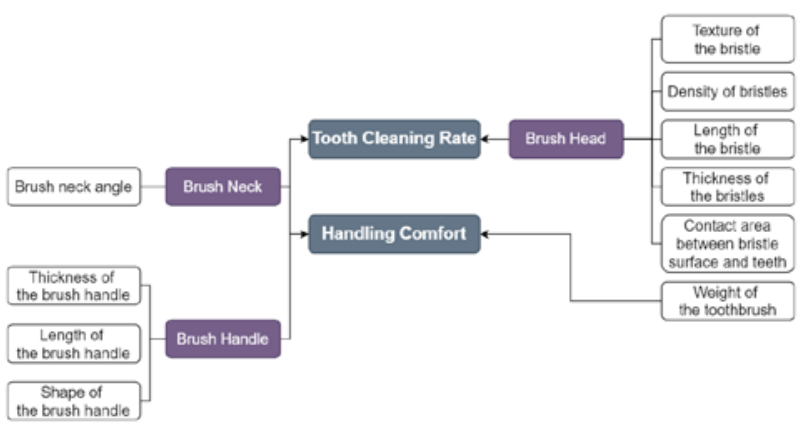

Figure 1. Factors affecting tooth cleaning rate and handling comfort

\section{MATERIALS AND METHODS}

\subsection{Experiment Participant}

Twenty breeders with dog-feeding experience, aged between 20 and 50 years, 10 males and 10 females, were screened to read and sign the informed consent form before the experiment. All study subjects had good compliance and no uncooperative or lost visitors.

\subsection{Experiment Toothbrush}

The test toothbrush is modified based on the commercially available nylon bristle toothbrush. As shown in Figure 2, the toothbrush is divided into three parts, namely the brush head, the brush neck and the brush handle. Cut and separated the brush head from the brush handle, and combines the self-made 3D column printing neck, which can adjust the angle in two segments to ensure that the other specifications are exactly the same except for the brush neck shape. In the straight state, the brush head length is $28 \mathrm{~mm}$, the brush neck length is $55 \mathrm{~mm}$ and the brush handle length is $100 \mathrm{~mm}$.
The test toothbrushes were divided into five groups: group A with a completely straight toothbrush; group B with 15 degree forward tilting angle at upper neck; group $\mathrm{C}$ with 15 degree backward tilting angle at upper neck; group D with 15 degree forward tilting angle at lower neck, while its upper neck is parallel to the brush handle; and group E with 15 degree backward tilting angle at lower neck, while its upper neck is parallel to the brush handle. The toothbrush model is shown in Figure 3.

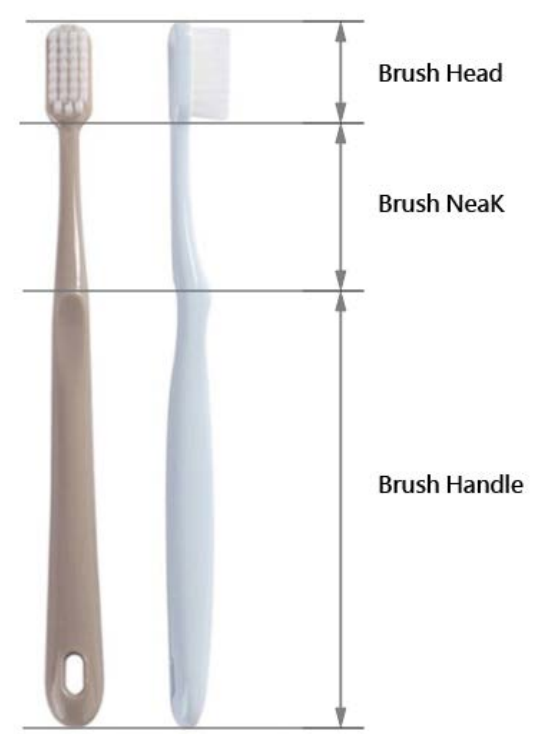

Figure 2. Toothbrush structure

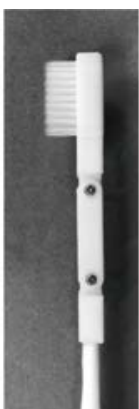

A

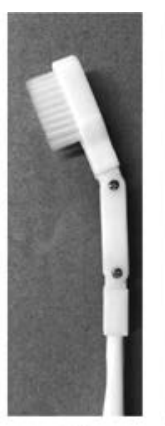

B

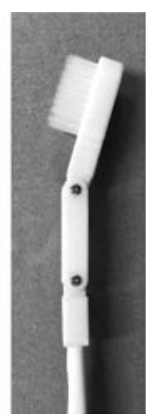

c

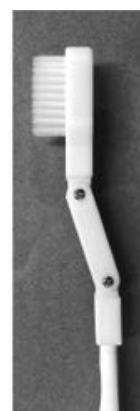

(1)

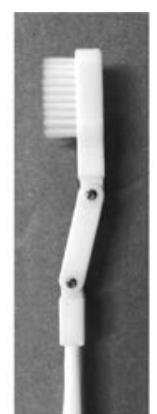

(e
Figure 3. Appearance of five groups of toothbrushes.

\subsection{Experimental Process}

The experiment was started by evenly coating the tooth surface of a medium-sized canine model with water powder pigment and allowing the pigment to dry completely. Volunteers were asked to perform the experiment by daily brushing their teeth for pet dogs, and five groups of toothbrushes were used for five trials, respectively, in a randomized order, with quantitative 20 $\mathrm{ml}$ of clear water to brush the teeth of this model for 3 minutes in each trial. (Figure 4) During tooth brushing, photographs and videos were taken to observe the changes in the operating posture and wrist angle of the subject. At the end of the five groups of tests, the subjects rated the operating comfort of each toothbrush in turn using a 5point Likert scale, with 5 being the most comfortable and 
1 being the most uncomfortable, with a brief explanation of the reasons.

\subsection{Experimental Data Collection}

At the end of each test, photographs were taken to record the incisors, canines and molars before and after tooth brushing. Pigment area on the tooth surface was calculated using ImageJ software. (Figure 5)

Tooth cleaning ratio $=($ pigment area before - pigment area after)/pigment area before.

\subsection{Statistical Analysis}

Statistical analysis was performed using SPSS software. The measurement data obeying the normal distribution by the normality test were expressed as $\bar{x} \pm \mathrm{s}$, and the single factor variation number analysis was performed for the tooth cleaning rate and operating comfort score.

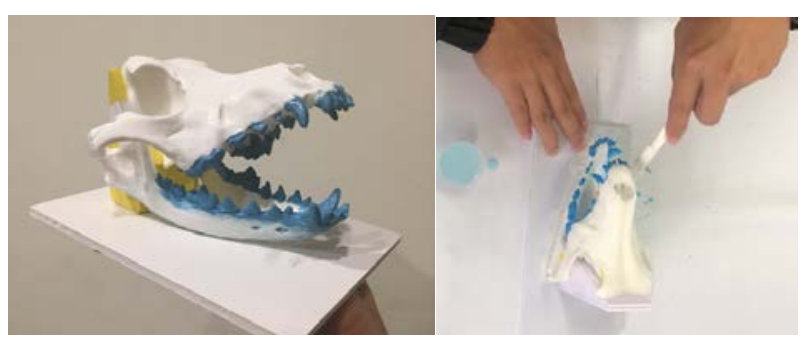

Figure 4. Medium canine tooth model and brush experimental process.

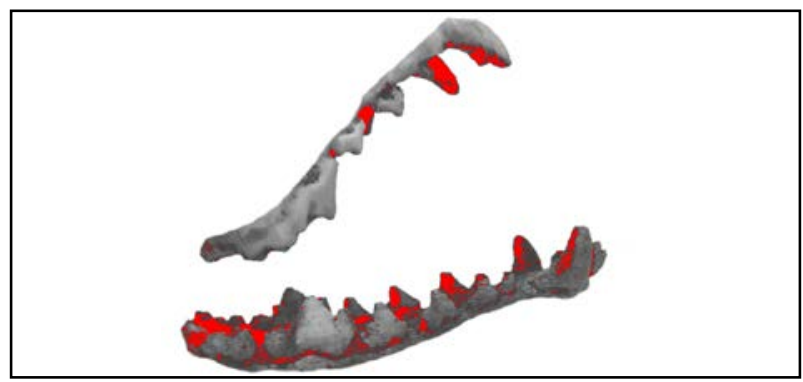

Figure 5. Residual pigment area calculated in ImageJ software.

\section{Prepare Your Paper Before Styling}

The differences in the tooth cleaning rates of incisors, canine teeth and molar teeth among the five groups had statistical significance $(\mathrm{F}=4.63 \sim 12.69, \mathrm{P}<0.05)$. Table 1 shows that group A were slightly better than the other groups in incisor cleaning; Table 2 shows that group $\mathrm{C}$ were the best in canine cleaning, followed by group D; and Table 3 shows that group $\mathrm{C}$ were significantly better than the other groups in molar cleaning. (Fig. $(1 \sim 3)$

There was significant difference in operation comfort ( $F=15.01, P<0.05)$, and the data showed that toothbrush operation of group D was the most comfortable. (Figure 4)
Table1. Comparison of pigment area and tooth cleaning rate on incisors before and after tooth brushing among five groups $(n=20)$

\begin{tabular}{lcll}
\hline Groups & $\begin{array}{l}\text { Pigment Area } \\
\text { Before Brushing }\end{array}$ & $\begin{array}{l}\text { Pigment Area } \\
\text { After } \\
\text { Brushing }\end{array}$ & $\begin{array}{l}\text { Tooth } \\
\text { Cleaning Rate } \\
(\chi / \%)\end{array}$ \\
\hline Group A & $324.80 \pm 18.61$ & $79.60 \pm 11.79$ & $75.49 \pm 3.38$ \\
Group B & $325.85 \pm 11.06$ & $\begin{array}{l}110.60 \\
\pm 14.65\end{array}$ & $65.81 \pm 5.75$ \\
& & $85.90 \pm 14.11$ & $73.48 \pm 4.25$ \\
Group C & $324.60 \pm 23.50$ & $92.60 \pm 15.90$ & $71.30 \pm 4.88$ \\
Group D & $323.15 \pm 19.00$ & $92.60 \pm 14.62$ & $72.78 \pm 4.35$ \\
Group E & $328.65 \pm 24.98$ & $89.25 \pm 14.62$ & \multicolumn{2}{c}{ Pigment } \\
\hline
\end{tabular}

Table2. Comparison of pigment area and tooth cleaning rate on canine teeth before and after tooth brushing among five groups $(n=20)$

\begin{tabular}{llll}
\hline Groups & $\begin{array}{l}\text { Pigment Area } \\
\text { Before Brushing }\end{array}$ & $\begin{array}{l}\text { Pigment Area } \\
\text { After } \\
\text { Brushing }\end{array}$ & $\begin{array}{l}\text { Tooth } \\
\text { Cleaning Rate } \\
(\chi / \%)\end{array}$ \\
\hline Group A & $273.60 \pm 16.17$ & $87.55 \pm 18.68$ & $68.03 \pm 6.32$ \\
Group B & $268.00 \pm 40.43$ & $95.35 \pm 19.54$ & $63.74 \pm 8.24$ \\
Group C & $283.35 \pm 23.02$ & $72.10 \pm 16.17$ & $74.41 \pm 5.76$ \\
Group D & $269.35 \pm 40.25$ & $80.50 \pm 15.94$ & $69.24 \pm 8.82$ \\
Group E & $279.40 \pm 16.45$ & $71.55 \pm 8.98$ & $74.33 \pm 3.28$ \\
\hline & & \multicolumn{3}{c}{ Pigment area unit: $\left(\mathrm{mm}^{2}\right)$}
\end{tabular}

Table3. Comparison of pigment area and tooth cleaning rate on molar teeth before and after tooth brushing among five groups $(n=20)$

\begin{tabular}{llll}
\hline Groups & $\begin{array}{l}\text { Pigment Area } \\
\text { Before Brushing }\end{array}$ & $\begin{array}{l}\text { Pigment Area } \\
\text { After Brushing }\end{array}$ & $\begin{array}{l}\text { Tooth } \\
\text { Cleaning Rate } \\
(\boldsymbol{\chi} / \%)\end{array}$ \\
\hline Group A & $1931.45 \pm 48.11$ & $664.05 \pm 116.50$ & $65.62 \pm 5.92$ \\
Group B & $1924.45 \pm 44.50$ & $769.10 \pm 171.494$ & $60.04 \pm 8.77$ \\
Group C & $1924.50 \pm 50.85$ & $571.60 \pm 141.31$ & $70.24 \pm 7.66$ \\
Group D & $1937.45 \pm 41.53$ & $679.80 \pm 133.44$ & $64.88 \pm 6.99$ \\
Group E & $1935.45 \pm 45.25$ & $689.85 \pm 165.93$ & $64.43 \pm 8.06$ \\
\hline & & \multicolumn{3}{c}{ Pigment area unit: $\left(\mathrm{mm}^{2}\right)$}
\end{tabular}

Table4. Comparison of operation comfort scores among five groups of toothbrushes $(n=20)$

\begin{tabular}{lll}
\hline Groups & Handling Comfort Score & Rank \\
\hline Group A & $3.30 \pm 0.57$ & 3 \\
Group B & $1.95 \pm 0.82$ & 5 \\
Group C & $3.55 \pm 1.09$ & 2 \\
Group D & $3.00 \pm 1.03$ & 4 \\
Group E & $3.80 \pm 0.69$ & 1 \\
\hline
\end{tabular}

\section{Discussion}

The purpose of this experiment was to verify whether cleaning efficacy of pet dog teeth and user comfortability can be improved by modifying the brush neck styles. Based on the results of the experiment, the toothbrush in group $\mathrm{C}$ showed the best tooth cleaning efficiency, while it only ranked second in the user comfortability score. Besides, two subjects stated after the experiment that the toothbrushes in group $\mathrm{C}$ made it easy to reach into the oral cavities and allow the bristles to adapt themselves to molar tooth surfaces. However, when cleaning lateral surfaces of the tooth, subjects' forearms had to be greatly expand and subjects felt tired after continuous brushing for a period of time. The authors of this paper believes that the retroversion angle can be appropriately reduced for effort 
saving in posterior molar cleaning. A forward-tilting brush head as in group B, often applied on the human toothbrushes, can reach deeper into the posterior molars when brushing user's own teeth. However, through the experimental observation, it is found that cleaning the ipsilateral teeth of the dominant hand produced a large amount of wrist dorsiflexion, and there was a radial deviation of the wrist when cleaning the teeth on nondominant-hand side, making the user uncomfortable, so the tooth cleaning efficiency and subjective comfortability are not ideal. In group D, the toothbrushes had feedbacks from three subjects, reflecting uncertainty on the forward tilting lower neck, which made it more difficult to aim the tooth to be brushed. Toothbrushes in group E had the highest comfortability scores, while there existed poor molar cleaning efficacy resulting from the interference by anterior teeth due to the backward tilting angle at lower neck.

In the past, the commonly used method to evaluate the cleaning effect of toothbrushes was to measure the PLI (plaque index) in the oral cavity [11], which means inspection with a probe combined with inspection. The probe is used to lightly scratch the tooth surface during inspection. The amount and thickness of the spots are scored. This method is difficult to implement for pet dogs, and the dog must be anesthetized before it can proceed smoothly. In this study, 3D printing technology was adopted to make tooth and toothbrush models, pigment was used to simulate dental plaque, and brushing activities of pet dogs were simulated. This method helped product testing proceed quickly and abundantly, shorten the product design and development cycle, and minimize external factors to strengthen the study. However, there are also limitations and slight unreality, and pet dogs' cooperation during tooth brushing is a well recognized problem for pet dog caretakers [12]. Besides, dogs' mouth flesh on both canine cheeks may block visibility during cleaning, and dog caretakers usually need to brush their teeth without seeing the posterior molars. To avoid the variation between the simulated experiment and the real situations, it is recommended to make more accurate product prototypes for further tests after the design direction is confirmed.

\section{Conclusion}

Nowadays, people's living standards are improving, and more and more attention is paid to the oral health of pet dogs. Using a toothbrush to clean the teeth of pet dogs is currently the most effective way to remove dental plaque. Studies have pointed out that oral hygiene is mainly controlled by the following three factors: the design of the toothbrush, the personal ability to use the toothbrush, and the frequency and length of the toothbrush. [13] Most manual toothbrushes on the market are composed of a brush head, a brush neck, and a brush handle. The design of these components will affect the removal rate of dental bacteria and the comfort of use. Among them, the toothbrush neck design can play a buffering effect during use, and the moderately curved shape of the neck ensures that the toothbrush head can penetrate into the mouth to the greatest extent and achieve the purpose of cleaning the mouth. The design of the neck brush shape will affect the posture during use and the movement of the toothbrush.

In the past, there were few studies on the cleaning tools of canine teeth. This article mainly focuses on the comparative study of the design of the neck brush of the pet dog toothbrush. And proposed an easy-to-operate test method for dog teeth cleaning tools. The method helps to identify a clear direction in early stage of the designing process. Based on the proposed method, the results obtained from the experiment show that the toothbrushes with backward tilting angle at their upper sections of neck are significantly better than the other four groups of toothbrushes in cleaning molars and canine teeth. In addition, when the lower section of brush neck tilts backward, and the upper section is parallel to the handle, a toothbrush is more likely to provide better comfortability to users. The author of this article suggests that when designing a dog's toothbrush, the front angle of brushing the neck can be tilted back, and that future research work can be fine-tuned for the tilting angle to achieve the best cleaning effect and comfort.

This article integrates tooth cleaning efficiency and ergonomic analysis, providing profound benefits in design and development of pet dog toothbrushes.

\section{References}

1. Wallis, C., \& Holcombe, L. J. (2020). A review of the frequency and impact of periodontal disease in dogs. Journal of Small Animal Practice, 61(9), 529-540. R. M. Allan,V. J. Adams,N. W. Johnston. Prospective randomised blinded clinical trial assessing effectiveness of three dental plaque control methods in dogs[J]. Journal of Small Animal Practice,2019,60(4).

2. Slot, D. E., Wiggelinkhuizen, L., Rosema, N. A. M., \& Van der Weijden, G. A. (2012). The efficacy of manual toothbrushes following a brushing exercise: a systematic review. International journal of dental hygiene, 10(3), 187-197.

3. Pretara-Spanedda P, Grossman E, Curro FA, Generallo C. Toothbrush bristle density: relationship to plaque removal. American Journal of Dentistry. 1989 Dec;2(6):345-348.

4. Beals D,Ngo T,Feng Y,Cook D,Grau D G,Weber D A. Development and laboratory evaluation of a new toothbrush with a novel brush head design.[J]. American journal of dentistry,2000,13(Spec No).

5. Cifcibasi, E., Koyuncuoglu, C. Z., Baser, U., Bozacioglu, B., Kasali, K., \& Cintan, S. (2014). Comparison of manual toothbrushes with different bristle designs in terms of cleaning efficacy and potential role on gingival recession. European journal of dentistry, 8(3), 395.

6. Beke, A. L. (1967). Functional stiffness characteristics of toothbrush bristles. Journal of dental research, 46(4), 666-671.

7. Thamke, M. V., Beldar, A., Thakkar, P., Murkute, S., Ranmare, V., \& Hudwekar, A. (2018). Comparison of 
bacterial contamination and antibacterial efficacy in bristles of charcoal toothbrushes versus noncharcoal toothbrushes: A microbiological study. Contemporary clinical dentistry, 9(3), 463.

8. Battaglia, A. (2008). The Bass technique using a specially designed toothbrush. International journal of dental hygiene, 6(3), 183-187.

9. Cheng, G., Li, D., Sun, X. F., \& Gong, P. (2011, September). The model research of upper limb and toothbrush in ergonomics. In 2011 IEEE 18th International Conference on Industrial Engineering and Engineering Management (pp. 644-646). IEEE.MA Hurairah, Z Ali, M Rasool. (2018). To Study the Anthropometry and Other Ergonomic Aspects of Toothbrush.

10. Zhu, Y. F. (2011). A Design Principle of Toothbrush Handle Based on Ergonomic Theory. Applied Mechanics and Materials, 121-126, 622-626.

11. Löe, H. (1967). The gingival index, the plaque index and the retention index systems. The Journal of Periodontology, 38(6), 610-616.

12. Miller, B. R., \& Harvey, C. E. (1994). Compliance with oral hygiene recommendations following periodontal treatment in client-owned dogs. Journal of Veterinary Dentistry, 11(1), 18-19.

13. Frendsen A. (1986). Mechanical oral hygiene practices. In: Loe H, Kleinman DV, eds. Dental Plaque Control Measures and Oral Hygiene Practice. Oxford, IRL Press, 93-116. 